\section{Finding the right questions to ask about the lives of human clones}

\section{Child-development experts may have useful information.}

Sir - I welcome Lee Silver's call in his Words essay "What are clones?" (Nature 412, 21; 2001) for an informed public debate on human reproductive cloning, but I question his proposed basis for the discussion. Silver concludes that a person produced by nuclear transfer would be "a unique and unpredictable child who had the same DNA sequence as someone else, but nothing more".

I disagree with his implication that a clone would necessarily have the same opportunity for individual development as a child produced by sexual reproduction. The reasons most commonly suggested for producing a clone are to overcome infertility or to replace a dead child. In the first case, the clone would be produced from one of the parents; in the second it would be from a child lost in an accident or after illness. The clone would be physically very similar to the original and have quite a similar personality, because of their shared inheritance. There would be greater similarity to the original in both regards than to any other person except an identical twin born at the same time as the original.

It seems inevitable that this unusual similarity and the reasons for the production of the clone would influence relationships formed by the child throughout its lifetime. If the original was a dead child in the same family, there is no doubt that the parents wish "to use cloning to bring dead children back to life", as noted by Silver. What then would be the effect - not only on parents, but also on relatives, friends, school teachers and other children - of expectations that the clone would grow up like the original? If a parent were the original, would they have unusual and unreasonable expectations as to how the clone should develop? As the parent aged, how would the cloned child then react to seeing its physical future?

It is concern over these issues that makes me and many others reject the suggestion of cloning a person. The views of those who have studied child development would be very welcome. Ian Wilmut

Roslin Institute, Roslin, Midlothian EH25 9PS, UK investigated and remedial measures sought. If scientists, journal editors and learned societies take the initiative in calling for such investigation, this objective will be achieved more quickly.

S. B. Vohora ${ }^{\star}$, Divya Vohora $\dagger$

${ }^{*}$ Department of Toxicology, Faculty of Science,

tDepartment of Pharmacology, Faculty of

Pharmacy,

Hamdard University, New Delhi 110 062, India

\section{Genome helpdesk site keeps information public}

Sir - The European Molecular Biology Laboratory (EMBL), with additional support from the UK Medical Research Council and the Wellcome Trust, has established a new genome helpdesk, http://www.ensembl.org, at the European Bioinformatics Institute (EBI). The EBI is the primary provider of public genomesequence data within Europe. The US National Institutes of Health has expressed its strong support for the EBI helpdesk, which will complement the existing service, http://www.ncbi.nlm.nih.gov/ genome/guide, at the US National Center for Biotechnology Information (NCBI).

Together, these initiatives will ensure that the vast potential of the publicly funded genome-sequence databases is fully exploited and freely available for all to use.

The NCBI helpdesk answers more than 300 queries each day from scientists and is Together with the new EBI site, users will have easy access to an unsurpassed collection of genome sequences and tools for their interpretation. Both helpdesks are staffed by expert teams and rapidly answer queries by e-mail as a public service, available without restriction. It adds to a range of resources provided freely by the EBI and NCBI for commercial and academic scientists to maximize the potential of the public genome databases. These resources are continuously being refined and improved as new genome data are added.

\section{Sir George Radda}

Medical Research Council, 20 Park Crescent, London W1B 1AL, UK

Other signatories to this letter:

Mike Dexter Wellcome Trust, London, UK Fotis Kafatos EMBL, Heidelberg, Germany Christian Bréchot INSERM, Paris, France

Philippe Kourilsky Institut Pasteur, Paris, France

Joachim Seelig Biozentrum, University of Basel, Switzerland Alan Bernstein Coalition For Biomedical \& Health Research, Ottawa, Canada

Motoya Katsuki National Institute for Basic Biology, Okazaki, Japan W. G. van Aken ZON NW (Council for Medical Research and Health Development), The Hague, Holland

E.-L. Winnacker DFG, Bonn, Germany

Heidi Diggelmann National Science Foundation, Zurich, Switzerland
Is the quality of our publications as poor as it seems? Are impact factors giving a true picture? The reasons for the situation in India must be properly \section{an invaluable guide for navigation of the publicly available genome databases. \\ National Scientific Documentation Cen} international journals. An outstanding piece of research published in a less wellknown journal might go unnoticed, restricting coverage or introducing an regional bias, for example between
developed and developing countries? The criteria for inclusion in SCI are not known. 